\title{
Different roles of Akt and mechanistic target of rapamycin in serum-dependent chondroprotection of human osteoarthritic chondrocytes
}

\author{
TONGEN ZHANG ${ }^{1}$, JIJIE LIU ${ }^{1}$, XINPENG ZHENG ${ }^{1}$, BING ZHANG $^{2}$ and CHUN XIA ${ }^{1}$ \\ ${ }^{1}$ Department of Sports Medicine and Joint Surgery, Zhongshan Hospital, Xiamen University, \\ Xiamen, Fujian 361004; ${ }^{2}$ Medical School, Xiamen University, Xiamen, Fujian 361102, P.R. China
}

Received March 24, 2017; Accepted November 22, 2017

DOI: $10.3892 /$ ijmm.2017.3285

\begin{abstract}
Despite various animal serums being used widely to culture chondrocytes, the regulatory mechanism of serum on chondrocyte activities has not been elucidated. In the present study, human osteoarthritis (OA) chondrocytes were used to perform in vitro investigations on the effect of different concentrations of bovine fetal serum on extracellular matrix synthesis, cell proliferation and autophagy using the Cell Counting Kit- 8 analysis, a laser-scanning confocal microscope, and western blot analysis. The results demonstrated that $5 \%$ serum exerted a chondroprotective effect more than the other concentrations of serum, as it simultaneously promoted cell proliferation, autophagy, and ECM synthesis in human OA chondrocytes. Furthermore, the decreased mechanistic target of rapamycin (mTOR) and increased Akt were observed in 5\% serum-treated OA chondrocytes. Either mTOR or Akt inhibitor influenced the effect of $5 \%$ serum on cell proliferation and autophagy in human OA chondrocytes, which was associated with LC-3B or B-cell lymphoma-2 (Bcl-2) signal molecules. Consistent with previous studies, the present study proposes that $5 \%$ serum promotes cell proliferation via the Akt/Bcl-2 axis and induces autophagy via the mTOR/LC-3B axis in human OA chondrocytes. Furthermore, the different roles of Akt and mTOR in the cell processes of human OA chondrocytes require consideration for preclinical and clinical therapy of OA.
\end{abstract}

Correspondence to: Professor Bing Zhang, Medical School, Xiamen University, 4221 Southern Road of Xiangan, Xiamen, Fujian 361102, P.R. China

E-mail: cristal66@xmu.edu.cn

Professor Chun Xia, Department of Sports Medicine and Joint Surgery, Zhongshan Hospital, Xiamen University, 201 Southern Road of Hubin, Xiamen, Fujian 361004, P.R. China

E-mail: chunxia@xmu.edu.cn

Key words: Akt, mechanistic target of rapamycin, serum-dependent chondroprotection, human osteoarthritic chondrocytes

\section{Introduction}

Osteoarthritis (OA) is the most prevalent degenerative disease of the articulating joints, and deterioration of cartilage extracellular matrix (ECM) homeostasis is one of its pathological characteristics. As the sole cell type in cartilage, chondrocytes are responsible for regulating ECM anabolism and catabolism. Therefore, chondrocyte activities, including autophagy, apoptosis and proliferation, are crucial for preserving cartilage homeostasis. For example, autophagy, a lysosomal degradation process, is essential for survival, differentiation, development and homeostasis (1). It has been reported to control the secretion of type II collagen ( $\mathrm{Col} 2)$, one of the primary components of cartilage ECM. In the absence of autophagy in growth-plate chondrocytes, $\mathrm{Col} 2$ decreased due to the abnormal accumulation of collagen molecules in the endoplasmic reticulum (2). Increased autophagy is an adaptive response to protect chondrocytes from stresses (3). In addition, the blockade of chondrocyte apoptosis and the promotion of chondrocyte proliferation may be potential therapeutic strategies to treat OA. Berberine may ameliorate cartilage degeneration from OA by promoting cell survival and matrix production of chondrocytes in interleukin (IL)-1 $\beta$-stimulated articular chondrocytes and in a rat OA model (4). Morroniside exerted chondroprotective effects on OA chondrocytes by promoting cell proliferation and ECM synthesis (5). Hyaluronic acid may suppress chondrocyte apoptosis in a dose-dependent manner in IL-1 $\beta$-induced OA, which may be an underlying mechanism of the clinical action of intra-articular injection of hyaluronic acid in the treatment of OA (6).

The majority of cell signaling molecules are involved in regulating OA chondrocyte autophagy, proliferation and apoptosis, including protein kinase $\mathrm{B}$ (PKB/Akt) and mechanistic target of rapamycin (mTOR) and extracellular signal-regulated kinase (ERK). For example, mTOR targeting prevents physical harm to joints in diabetic mice by autophagy activation (7). Cartilage-specific deletion of mTOR upregulates autophagy and protects mice from OA (8). Akt and ERK contribute to the chondroprotective effect of morroniside on OA chondrocytes by promoting chondrocyte proliferation and ECM synthesis $(5,9,10)$. Therefore, present study predominantly focuses on elucidating the role and regulatory mechanism of 
complicated cell signaling molecules involved in chondrocyte events.

Various animal serums are added to chondrocyte culture media as they contain numerous nutrient components and cytokines. Notably, in the mid-1990s, a novel therapeutic strategy, the intra-articular injection of autologous conditioned serum (ACS; Orthokine) was developed, and has recently been demonstrated to be safe and effective in a number of clinical studies, and is widely adopted in Europe $(11,12)$. ACS contains enriched anti-inflammatory cytokines, including interleukin (IL)-1Ra, IL-10 and IL-13, and various growth factors, while maintaining low concentrations of pro-inflammatory cytokines, such as IL-1 $\beta$ and tumor necrosis factor (TNF)- $\alpha$. However, the elevated concentrations of pro-inflammatory cytokines in ACS have no adverse effects on proteoglycan turnover in the cartilage explant cultures $(12,13)$. As a result, understanding the regulatory mechanism of serum is needed to elucidate its effects on OA therapy.

The present study aims to evaluate the effect of different types of bovine fetal serum on human OA chondrocyte proliferation, autophagy and ECM metabolism, and to further investigate the regulatory mechanism associated with Akt and mTOR signaling molecules.

\section{Materials and methods}

Reagents and antibodies. Inhibitors [mTOR inhibitor Rapamycin (cat. no. 9904) and PI3K/Akt inhibitor LY294002 (cat. no. 9901)] and antibodies against mTOR (cat. no. 2983), phosphorylated (p)-mTOR (Ser2448; cat. on. 2971), Akt (cat. no. 4691), p-Akt (Ser473; cat. no. 4051), B-cell lymphoma-2 (Bcl-2, cat. no. 2870), p-S6 (Ser235/236; cat. no. 2211), Beclin 1 (cat. no. 4122), AMP-activated protein kinase (AMPK) $\alpha$ (cat. no. 5831), p-AMPK $\alpha$ (Thr172; cat. no. 2535) and LC-3B (cat. no. 3868) were purchased from Cell Signaling Technology Inc. (Danvers, MA, USA). Anti-GAPDH antibody (cat. no. 0411) was purchased from Santa Cruz Biotechnology, Inc. (Dallas, TX, USA). Antibodies targeting Col 2 (cat. no. WH0001280M1) and aggrecan (cat. no. SAB4500662) were purchased from Sigma-Aldrich (Merck KGaA, Darmstadt, Germany). Anti-proliferating cell nuclear antigen antibody (cat. no. BM0104) was purchased from Wuhan Boster Biological Technology., Ltd. (Wuhan, Hubei, China). Secondary antibodies (goat anti-mouse; cat. no. SA00001-1; and goat anti-rabbit; cat. no. SA00001-2) were purchased from Proteintech Group, Inc. (Chicago, IL, USA). Other commercially available reagents of the highest grade were obtained.

Human OA chondrocyte isolation and culture. Subsequent to receiving all patient consent and, in accordance with the ethical guidelines of Zhongshan Hospital of Xiamen University (Xiamen, China), human OA articular cartilage was obtained from advanced OA patients who were undergoing total knee replacement surgery in Zhongshan Hospital of Xiamen University (Xiamen, China). Under sterile conditions, the articular cartilage on the femoral condyle and tibial plateau was minced into small pieces and digested with $0.2 \%$ type II collagenase as described previously $(14,15)$. The isolated chondrocytes were cultured in Dulbecco's modified
Eagle's medium/F12 medium with $10 \%$ fetal bovine serum (FBS; v/v) plus $1 \%$ penicillin/streptomycin at $37^{\circ} \mathrm{C}$ for $72 \mathrm{~h}$ in a water-saturated atmosphere of $5 \% \mathrm{CO}_{2}$. When chondrocytes were grown to $70-80 \%$ confluence, the cells were plated in $60-\mathrm{mm}$ Petri dishes $\left(2 \times 10^{6}\right.$ cells/dish) or 96 -well plates ( $3 \times 10^{4}$ cells/well), followed by the evaluation of chondrocytes using immunohistological techniques and treatment with or without different inhibitors. Ethical approval for the study was obtained from the Ethics Committee of the Medical School, Xiamen University (Xiamen, China).

Cell viability assay. Cells were plated in 96-well plates $\left(2 \times 10^{5}\right.$ cells/well) and starved with serum-free medium for $24 \mathrm{~h}$. The medium was then replaced with fresh medium with different concentrations of serum in the presence or absence of inhibitors for a further $24 \mathrm{~h}$. The Cell Counting Kit-8 (CCK8) assay (cat. no. C0037; Beyotime Institute of Biotechnology, Haimen, China) was then performed, as described previously (16). The end product was quantified spectrophotometrically at a wavelength of $450 \mathrm{~nm}$. The optical density values correspond to the number of viable cells (17).

Western blot analysis. Cells were collected by centrifugation ( $825 \mathrm{x} \mathrm{g}, 7 \mathrm{~min}$ ) at room temperature and lysed in Radioimmunoprecipitation assay lysis buffer containing $1 \%$ Triton X-100, $1 \%$ deoxycholate and $0.1 \%$ SDS (premixed solution; cat. no. P0013B, Byeotime Institute of Biotechnology). Lysates were then centrifuged at $19,071 \mathrm{x}$ g or $30 \mathrm{~min}$ at $4^{\circ} \mathrm{C}$ and the protein concentration was determined using a Pierce Bicinchoninic acid Protein assay kit (Thermo Fisher Scientific, Inc., Waltham, MA, USA). Protein extracts were subjected to SDS-PAGE (8-12\% according to the molecular weight of the protein) and transferred to polyvinylidene fluoride membranes (60 min, $100 \mathrm{~V}$; EMD Millipore, Billerica, MA, USA) as described previously (18). The membranes were incubated with the indicated primary antibodies at $4^{\circ} \mathrm{C}$ overnight and corresponding secondary antibodies at room temperature for 1-2 $\mathrm{h}$ in succession. The signal was monitored using a chemiluminescent detection system according to the manufacturer's protocol (cat. no. WBKLS0500; EMD Millipore).

Laser-scanning confocal microscope. Chondrocytes were fixed in $4 \%$ paraformaldehyde after harvesting. For staining of the endogenous LC-3B proteins, the cells were incubated with anti-LC-3B antibody (dilution, 1:200) followed by Cy3-conjugated secondary antibody (Merck KGaA) as described previously (18). The cells were stained with $50 \mathrm{mg} / \mathrm{ml}$ 4',6-diamidino-2-phenylindole (Sigma-Aldrich; Merck KGaA) to visualize the nuclei simultaneously at room temperature for 5 min (18). The stained cells were finally visualized under a confocal microscope (Leica TCS SP2 SE; Leica Microsystems $\mathrm{GmbH}$, Wetzlar, Germany).

Statistical analysis. Experimental data were formulated as the means \pm standard error of the mean of three independent samples. Statistical analyses were performed with GraphPad Prism 5 software (GraphPad Software, Inc., La Jolla, CA, USA), using one-way ANOVA. $\mathrm{P}<0.05$ was considered to indicate a statistically significant difference. 




Figure 1. Effect of different concentrations of serum on cell proliferation in human OA chondrocytes. Cultured human OA chondrocytes were starved for $24 \mathrm{~h}$ and treated with different concentrations of serum (1,5,10 and 20\%) for $24 \mathrm{~h}$. (A) Cell viability was evaluated using a Cell Counting Kit 8 assay. (B) The expression levels of PCNA, p-S6, and Bcl-2 were detected by western blotting. The blots were normalized to an endogenous protein (GAPDH). (C) The expression levels of aggrecan and $\mathrm{Col} 2$ were detected with western blotting. The blots were normalized to an endogenous protein (GAPDH). The data are representative of three independent experiments, each yielding similar results $\left({ }^{*} \mathrm{P}<0.05,{ }^{* *} \mathrm{P}<0.01\right.$ and ${ }^{* * * *} \mathrm{P}<0.001$ vs. serum-free group). OA, osteoarthritis; PCNA, proliferation cell nuclear antigen; p-, phosphorylated; Bcl-2, B-cell lymphoma 2; Col 2, type II collagen.

\section{Results}

Effect of different concentrations of serum on cell proliferation and ECM synthesis in human OA chondrocytes. To investigate the effect of serum on OA chondrocyte proliferation, cultured human OA chondrocytes were starved for $24 \mathrm{~h}$ and treated with different concentrations of serum $(1,5,10$ and 20\%) for $24 \mathrm{~h}$. The cell viability was measured using a CCK 8 assay. Fig. 1A indicates that chondrocyte viability significantly increased in 5 and 20\% serum-treated groups without any alteration observed in the other groups $(\mathrm{P}<0.05$ vs. the serum-free group). Proliferation cell nuclear antigen (PCNA), Bcl-2 and S6 are predominant biomarkers of cell proliferation, and their expression levels were detected by western blotting. PCNA, Bcl-2 p-S6 expression levels increased in the 5\% serum-treated group (Fig. 1B; $\mathrm{P}<0.05$ vs. the serum-free group). 1,10 or $20 \%$ serum led to increased Bcl-2 and p-S6 expression levels (Fig. 1B, $\mathrm{P}<0.05$ vs. the serum-free group). In addition, the expression levels of the predominant components of the ECM, aggrecan and Col 2, were detected by western blotting analysis. The 5\% serum simultaneously enhanced aggrecan and $\mathrm{Col} 2$ expression levels in the human OA chondrocytes (Fig. 1C; $\mathrm{P}<0.05$ vs. the serum-free group). The $10 \%$ serum only enhanced the expression level of $\mathrm{Col} 2$ (Fig. 1C; $\mathrm{P}<0.05$ vs. the serum-free group). Consequently, only $5 \%$ serum simultaneously significantly enhanced cell viability along with the increase in PCNA, Bcl-2 and p-S6 expression levels in human OA chondrocytes, and promoted ECM synthesis via the increase of aggrecan and $\mathrm{Col} 2$ expression levels in human OA chondrocytes.

Effect of different concentrations of serum on autophagy in human OA chondrocytes. As autophagy is essential for survival, differentiation, development and homeostasis (1), the present study determined whether serum may regulate autophagy of OA chondrocytes. Cultured human OA chondrocytes were starved for $24 \mathrm{~h}$ and treated with different concentrations of serum $(1,5,10$ and $20 \%)$ for $4 \mathrm{~h}$. These autophagy-associated markers, including LC-3B, Beclin 1 and AMPK $\alpha$, were detected by western blotting. Fig. 2A demonstrated that LC-3B II, Beclin 1 and p-AMPK $\alpha$ expression levels increased in the $5 \%$ serum-treated group $(\mathrm{P}<0.05$ vs. the serum-free group). The $1 \%$ serum led to increased LC-3B II and p-AMPK $\alpha$ expression levels. Additionally, the results of laser-scanning confocal microscopy demonstrated that the endogenous LC-3B expression level with anti-LC-3B antibody in 5\% serum-treated OA chondrocytes was higher than the other concentration serum-treated groups, compared with the serum-free group (Fig. 2B). Overall, it was identified that only $5 \%$ serum could simultaneously enhance the expression levels of LC-3B, Beclin 1 and p-AMPK $\alpha$ in human OA chondrocytes.

Akt and mTOR participate in regulating cell proliferation and autophagy in 5\% serum-treated human OA chondrocytes. Akt and mTOR have been implicated in OA pathogenesis; thus, the phosphorylation levels of mTOR and Akt were investigated in different concentrations of serum-treated human OA chondrocytes. Fig. $3 \mathrm{~A}$ demonstrates that $5 \%$ serum caused a significant decrease in p-mTOR andincrease in p-Akt, while the other concentrationss did not alter the p-mTOR and $\mathrm{p}$-Akt expression levels $(\mathrm{P}<0.05$ vs. the serum-free group). To further investigate the role of mTOR and Akt on cellular proliferation and autophagy in 5\% serum-treated human OA chondrocytes, the starved cells were treated with $50 \mathrm{nM}$ rapamycin (mTOR inhibitor) or $10 \mathrm{mM} \mathrm{LY} 294002$ (phosphoinositide 3-kinase; PI3K/Akt inhibitor) for $2 \mathrm{~h}$, 


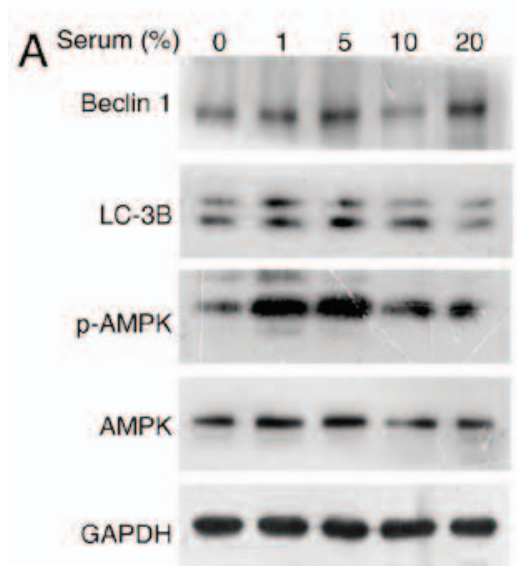

B Serum (\%)
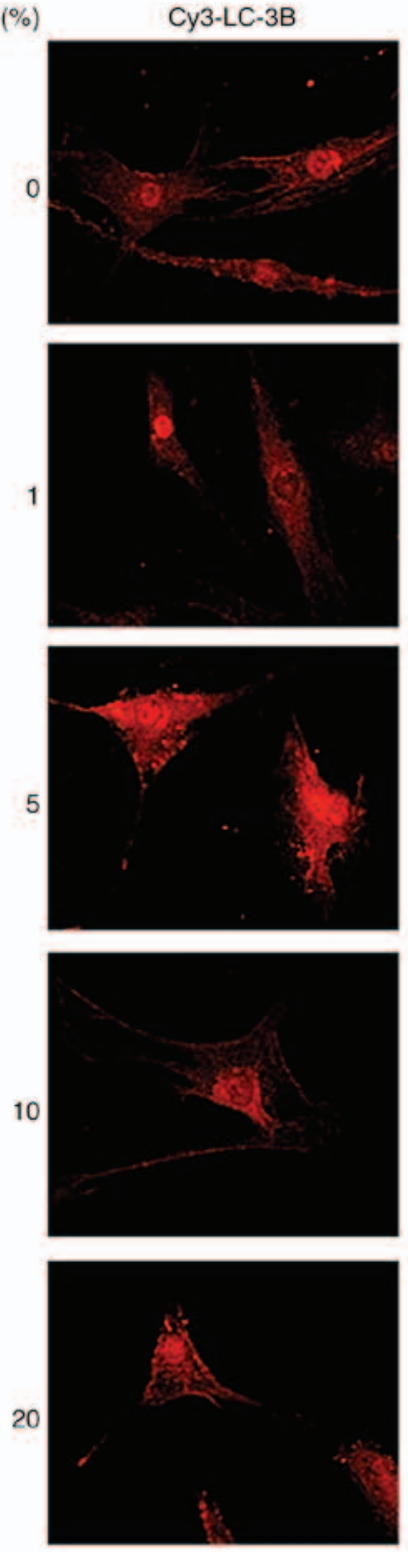
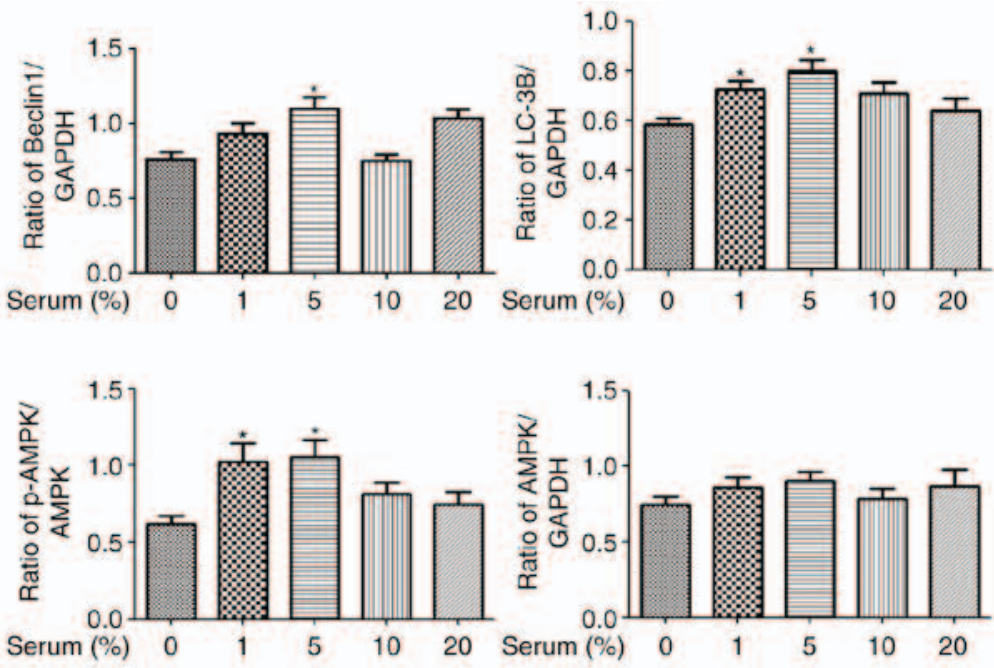

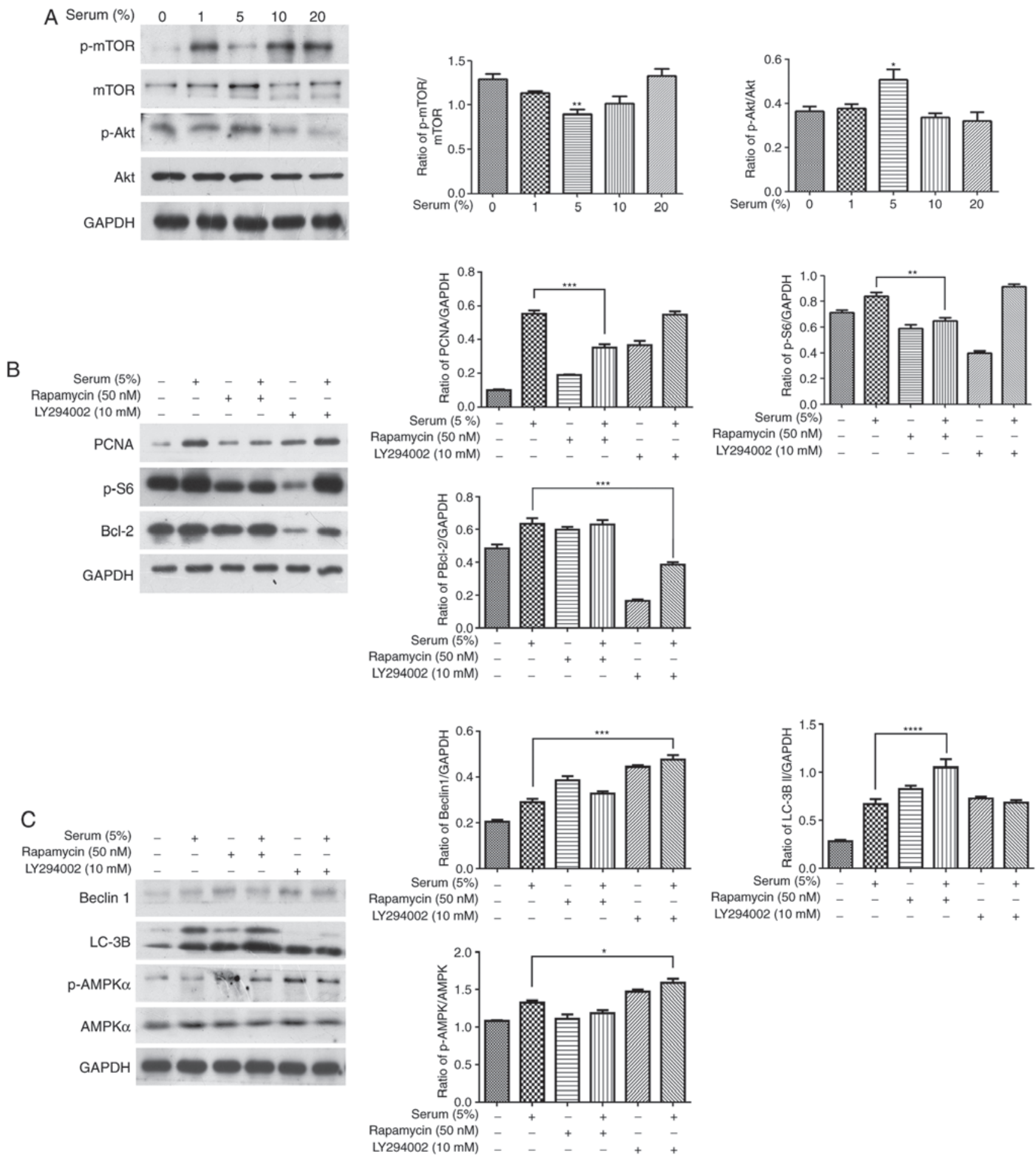

Figure 3. Effect of Akt and mTOR inhibitors on serum-associated cell proliferation and autophagy in human OA chondrocytes. (A) Cultured human OA chondrocytes were starved for $24 \mathrm{~h}$ and treated with different concentrations of serum (1, 5, 10 and 20\%) for $24 \mathrm{~h}$. The expression levels of Akt, p-Akt, mTOR and p-mTOR were detected with western blotting. (B and C) The starved chondrocytes were pretreated with $50 \mathrm{nM}$ rapamycin (mTOR inhibitor) or $10 \mathrm{mM} \mathrm{LY} 294002$ (PI3K/Akt inhibitor) for $2 \mathrm{~h}$, respectively, followed with 5\% serum for $4 \mathrm{~h}$. The expression levels of PCNA, p-S6, Bcl-2, Beclin 1, LC-3B, p-AMPK $\alpha$ and AMPK $\alpha$ were detected by western blotting. The blots were normalized to an endogenous protein (GAPDH). The data are representative of three independent experiments, each yielding similar results $\left({ }^{*} \mathrm{P}<0.05,{ }^{* *} \mathrm{P}<0.01,{ }^{* * *} \mathrm{P}<0.001\right.$ and ${ }^{* * * * *} \mathrm{P}<0.0001$ vs. serum-free group or untreated $5 \%$ serum-treated group). mTOR, mechanistic target of rapamycin; OA, osteoarthritis; p-, phosphorylated; PI3K, phosphoinositide 3-kinase; PCNA, proliferation cell nuclear antigen; Bcl-2, B-cell lymphoma 2.

respectively. This was followed by $5 \%$ serum treatment at $37^{\circ} \mathrm{C}$ for $4 \mathrm{~h}$ in a water-saturated atmosphere of $5 \% \mathrm{CO}_{2}$. The results of Fig. 3B demonstrate that rapamycin reduced PCNA and p-S6 expression levels in the 5\% serum-treated group ( $\mathrm{P}<0.01$ vs. the untreated $5 \%$ serum-treated group), while the Bcl-2 expression level did not significantly change. LY294002 significantly inhibited the level of Bcl-2 expression in the 5\% serum-treated groups without any alterations to the PCNA and p-S6 expression levels (Fig. 3B; $\mathrm{P}<0.001$ vs. the untreated 5\% serum-treated group). Furthermore, Fig. 3C 


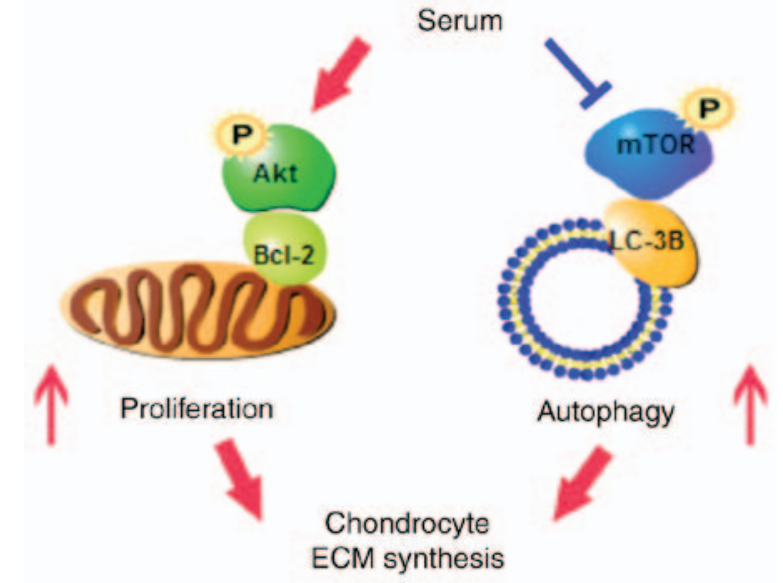

Figure 4. Proposed model of serum-dependent chondroprotection associated with Akt and mTOR. mTOR, mechanistic target of rapamycin; ECM, extracellular matrix; Bcl-2, B-cell lymphoma 2; p, phosphorylated.

demonstrated that rapamycin led to the increase in LC-3B II expression level in the 5\% serum-treated group without any alteration to the p-AMPK $\alpha$ and Beclin 1 expression levels $(\mathrm{P}<0.0001$ vs. the untreated $5 \%$ serum-treated group). Beclin 1 and p-AMPK $\alpha$ expression levels increased in the LY294002-treated 5\% serum-treated group, while LC-3B II did not significantly change $(\mathrm{P}<0.01$ vs. the untreated $5 \%$ serum-treated group). Therefore, the addition of either rapamycin or LY294002 modulated cell proliferation and autophagy in human OA chondrocytes, by targeting different signaling molecules.

\section{Discussion}

In the present study, the effect of different concentrations of serum on cell events was investigated in human OA chondrocytes. It is demonstrated that only $5 \%$ serum was able to simultaneously regulate cell proliferation, ECM synthesis and autophagy induction in human OA chondrocytes, compared with the other concentrations. Notably, decreased mTOR and increased Akt expression levels were observed in the 5\% serum-treated group. Furthermore, either mTOR or Akt inhibition modulated cell proliferation and autophagy by targeting different signaling molecules in human OA chondrocytes. Combined with the findings of previous studies, it is proposed that $5 \%$ serum promotes cell proliferation via the Akt/Bcl-2 axis and induces autophagy via the mTOR/LC3B axis in human OA chondrocytes.

Although $10 \%$ serum is always used to culture chondrocytes, certain studies indicate that chondrocytes cultured with serum-free medium exhibit no proliferation difference when compared with those cultured with $10 \%$ FBS (19). Human nasal chondrocytes proliferate at comparable rates in different serum conditions, with no statistically significant differences observed between the lower percentage of autologous serum (2\%) and $10 \%$ FBS (20). In the present study, treatment with $5 \%$ serum, not $10 \%$, simultaneously resulted in increased cell proliferation, autophagy and ECM synthesis in human OA chondrocytes. Therefore, it is proposed that $5 \%$ serum may be more suitable for culturing chondrocytes than $10 \%$ serum, at least in human OA chondrocytes.
Under high nutrient conditions, mTOR suppresses cell autophagy by phosphorylating autophagy related 13 (Atg13) to prevent the interaction between Atg13 and unc-51 like autophagy activating kinase (ULK), or phosphorylating ULK Ser757 to disrupt the interaction between AMPK and ULK (21). Conversely, activated mTORC1, due to the inactivation of TSC complex subunit 2 with Akt, triggers the mTOR/S6 axis to regulate protein synthesis, promoting cell proliferation (22-24). Therefore, activated mTOR may perform different functions in cellular processes, including promoting cell proliferation and suppressing autophagy induction. In the present study, the cell viability, PCNA, Bcl-2, aggrecan and Col 2 expression levels increased in the 5\% serum-treated group in Fig. 1, indicating that $5 \%$ serum may promote cell proliferation and ECM synthesis in human OA chondrocytes. However, 5\% serum led to a significantly decreased p-mTOR expression level, implying that mTOR/S6-associated proliferation may be blocked and that decreased levels of mTOR expression, by 5\% serum, did not inhibit cell proliferation. By contrast, the increase of LC-3B II, Beclin 1 and p-AMPK $\alpha$ expression levels in the $5 \%$ serum-treated group indicated that $5 \%$ serum may induce autophagy. Furthermore, the inhibitor of mTOR caused a significant increase in LC-3B II expression levels, demonstrating that decreased mTOR in 5\% serum-treated chondrocytes may be involved in autophagy induction of OA chondrocytes. Thus, mTOR may be involved in autophagy induction in 5\% serum-treated human OA chondrocytes, but not cell proliferation. The regulation of mTOR on chondrocyte autophagy is well known. Chondrocyte autophagy is stimulated by hypoxia-inducible factor-1-dependent AMPK activation and mTOR suppression (25). Inhibition of the $\mathrm{PI} 3 \mathrm{~K} / \mathrm{AKT} / \mathrm{mTOR}$ signaling pathway can promote autophagy of articular chondrocytes and attenuate inflammatory response in rats with OA (26). The intra-articular injection of rapamycin, an mTOR inhibitor, reduced mTOR expression levels, leading to a delay in articular cartilage degradation in an OA murine model (27). Therefore, the present study proposed that mTOR may predominantly be involved in regulating autophagy induction in 5\% serum-treated chondrocytes.

The activation of Akt increases in normal chondrocytes more than in OA chondrocytes, and is required for basal and insulin-induced Col 2 expression in chondrocytes (28). Elevated production of tribbles pseudokinase 3, an inhibitor of Akt activation, has been reported to increase in OA chondrocytes (29). In addition, previous studies demonstrated that increased Akt expression levels by extracellular stimuli may protect articular cartilage from OA deterioration by promoting cell survival in human or rat OA chondrocytes $(4,5)$. Thus, Akt is a positive element for chondroprotection. Consistent with these studies, $5 \%$ serum significantly enhanced $\mathrm{p}$-Akt expression levels concomitantly with $\mathrm{Bcl}-2$ depression caused by LY294002, exhibiting the promoting effect of Akt on serum-associated cell proliferation of human OA chondrocytes. Therefore, $5 \%$ serum is hypothesized to promote cell proliferation, to a lesser extent, by triggering the Akt/Bcl-2 signal axis. However, Hahn-Windgassen et al (30) demonstrated that Akt is a negative regulator of AMPK. Metformin, an AMPK activator, decreased the phosphorylation of Akt in 
bladder cancer cells (31). Notably, Galasso et al (32) reported that in OA human cartilage, increased phosphatase and tensin homolog (Akt inhibitor), AMPK and autophagy reflected the chondrocyte responses observed during starvation and steroid depletion (32). The present data that LY294002 may upregulate p-AMPK $\alpha$ in 5\% serum-treated human OA chondrocytes was consistent with the above-mentioned studies. Therefore, Akt may reduce autophagy induction by suppressing $\mathrm{p}-\mathrm{AMPK} \alpha$. However, the induced autophagy of chondrocytes eventually increased in the 5\% serum-treated group, implying that increased Akt may predominantly promote proliferation, rather than exert inhibitory effects on autophagy induction in 5\% serum-treated human OA chondrocytes, which may be due to being interfered with by other signaling pathways, such as the $\mathrm{mTOR} / \mathrm{LC}-3 \mathrm{~B}$ axis.

The PI3K/Akt signaling pathway is an important activator of mTOR, although Akt-independent regulation of mTOR in response to mitogens, nutrient availability and conditions that deplete intracellular energy in cancer have been reported (33). Multiple signaling pathways are involved in regulating mTOR independently of Akt, such as LKB1/AMPK, AMPK/mTORC1, and calmodulin-dependent protein kinase kinase $\beta / A M P K$ signaling pathways (33). Furthermore, mTOR inhibition may result in feedback activation of Akt in cancer (33-35), consistent with the present data of the opposite effect of 5\% serum on Akt and mTOR in human OA chondrocytes (data not shown). Future studies are required to determine the interaction between Akt and mTOR on cell processes in 5\% serum-treated human OA chondrocytes.

In conclusion, 5\% serum may simultaneously promote cell proliferation and autophagy induction of OA chondrocytes with the increase of ECM synthesis by upregulating Akt and downregulating mTOR signaling molecules. Furthermore, decreased mTOR expression levels promoted autophagy induction in human OA chondrocytes via the mTOR/LC-3B axis, and increased Akt enhanced cell proliferation via the Akt/Bcl-2 axis (Fig. 4). Consequently, the effect of increased Akt and decreased mTOR expression levels on human OA chondrocytes should be considered simultaneously for the preclinical and clinical treatment of OA.

\section{Acknowledgements}

The authors would like to thank Dr Honghai Zhao and Dr Shaojie Wang (Zhongshan Hospital, Xiamen University) for supplying the materials in the manuscript. The present study was supported by the National Science Foundation of China (grant nos. 81572189 and 81371952) and the Natural Science Foundation of Xiamen, China (grant no. 3502Z20159014).

\section{References}

1. Levine B and Kroemer G: Autophagy in the pathogenesis of disease. Cell 132: 27-42, 2008.

2. Cinque L, Forrester A, Bartolomeo R, Svelto M, Venditti R, Montefusco S, Polishchuk E, Nusco E, Rossi A, Medina DL, et al: FGF signalling regulates bone growth through autophagy. Nature 528: 272-275, 2015.

3. SasakiH,TakayamaK,MatsushitaT,IshidaK,KuboS,MatsumotoT, Fujita N, Oka S, Kurosaka M and Kuroda R: Autophagy modulates osteoarthritis-related gene expression in human chondrocytes. Arthritis Rheum 64: 1920-1928, 2012.
4. Zhao H, Zhang T, Xia C, Shi L, Wang S, Zheng X, Hu T and Zhang B: Berberine ameliorates cartilage degeneration in interleukin-1 $\beta$-stimulated rat chondrocytes and in a rat model of osteoarthritis via Akt signalling. J Cell Mol Med 18: 283-292, 2014.

5. Cheng L, Zeng G, Liu Z, Zhang B, Cui X, Zhao H, Zheng X, Song $\mathrm{G}$, Kang $\mathrm{J}$ and $\mathrm{Xia}$ C: Protein kinase B and extracellular signal-regulated kinase contribute to the chondroprotective effect of morroniside on osteoarthritis chondrocytes. J Cell Mol Med 19: 1877-1886, 2015.

6. Zhou PH, Liu SQ and Peng H: The effect of hyaluronic acid on IL-1beta-induced chondrocyte apoptosis in a rat model of osteoarthritis. J Orthop Res 26: 1643-1648, 2008.

7. Ribeiro M, López de Figueroa P, Nogueira-Recalde U, Centeno A, Mendes AF, Blanco FJ and Caramés B: Diabetes-accelerated experimental osteoarthritis is prevented by autophagy activation. Osteoarthritis Cartilage 24: 2116-2125, 2016.

8. Zhang Y, Vasheghani F, Li YH, Blati M, Simeone K, Fahmi H, Lussier B, Roughley P, Lagares D, Pelletier JP, et al: Cartilage-specific deletion of mTOR upregulates autophagy and protects mice from osteoarthritis. Ann Rheum Dis 74: 1432-1440, 2015.

9. Cui X, Wang S, Cai H, Lin Y, Zheng X, Zhang B and Xia C: Overexpression of microRNA-634 suppresses survival and matrix synthesis of human osteoarthritis chondrocytes by targeting PIK3R1. Sci Rep 6: 23117, 2016.

10. Chen J, Crawford R and Xiao Y: Vertical inhibition of the $\mathrm{PI} 3 \mathrm{~K} / \mathrm{Akt} / \mathrm{mTOR}$ pathway for the treatment of osteoarthritis. J Cell Biochem 114: 245-249, 2013.

11. Baltzer AW, Moser C, Jansen SA and Krauspe R: Autologous conditioned serum (Orthokine) is an effective treatment for knee osteoarthritis. Osteoarthritis Cartilage 17: 152-160, 2009.

12. Moser C: Response to: cytokine profile of autologous conditioned serum for treatment of osteoarthritis, in vitro effects on cartilage metabolism and intra-articular levels after injection. Arthritis Res Ther 12: 410, 2010.

13. Rutgers M, Saris DB, Dhert WJ and Creemers LB: Cytokine profile of autologous conditioned serum for treatment of osteoarthritis, in vitro effects on cartilage metabolism and intra-articular levels after injection. Arthritis Res Ther 12: R114, 2010.

14. Attur MG, Dave M, Cipolletta C, Kang P, Goldring MB, Patel IR, Abramson SB and Amin AR: Reversal of autocrine and paracrine effects of interleukin 1 (IL-1) in human arthritis by type II IL-1 decoy receptor. Potential for pharmacological intervention. J Biol Chem 275: 40307-40315, 2000.

15. Zeng G, Cui X, Liu Z, Zhao H, Zheng X, Zhang B and Xia C: Disruption of phosphoinositide-specific phospholipases $C \gamma 1$ contributes to extracellular matrix synthesis of human osteoarthritis chondrocytes. Int J Mol Sci 15: 13236-13246, 2014

16. Li C, Cai S, Wang X and Jiang Z: Identification and characterization of ANO9 in stage II and III colorectal carcinoma. Oncotarget 6: 29324-29334, 2015.

17. Zheng $\mathrm{CH}$ and Levenston ME: Fact versus artifact: Avoiding erroneous estimates of sulfated glycosaminoglycan content using the dimethylmethylene blue colorimetric assay for tissue-engineered constructs. Eur Cell Mater 29: 224-236, 2015.

18. Zhang B and Xia C: 12-O-tetradecanoylphorbol-1, 3-acetate induces the negative regulation of protein kinase $\mathrm{B}$ by protein kinase Calpha during gastric cancer cell apoptosis. Cell Mol Biol Lett 15: 377-394, 2010.

19. Shao XX, Duncan NA, Lin L, Fu X, Zhang JY and Yu CL: Serum-free media for articular chondrocytes in vitro expansion. Chin Med J (Engl) 126: 2523-2529, 2013.

20. Wolf F, Haug M, Farhadi J, Candrian C, Martin I and Barbero A: A low percentage of autologous serum can replace bovine serum to engineer human nasal cartilage. Eur Cell Mater 15: 1-10, 2008.

21. Jain MV,Paczulla AM,Klonisch T,DimgbaFN, Rao SB, Roberg K, Schweizer F, Lengerke C, Davoodpour P, Palicharla VR, et al: Interconnections between apoptotic, autophagic and necrotic pathways: Implications for cancer therapy development. J Cell Mol Med 17: 12-29, 2013.

22. Laplante M and Sabatini DM: mTOR signaling in growth control and disease. Cell 149: 274-293, 2012.

23. Inoki $\mathrm{K}, \mathrm{Li} \mathrm{Y}, \mathrm{Xu} \mathrm{T}$ and Guan KL: Rheb GTPase is a direct target of TSC2 GAP activity and regulates mTOR signaling. Genes Dev 17: 1829-1834, 2003.

24. Tee AR, Manning BD, Roux PP, Cantley LC and Blenis J: Tuberous sclerosis complex gene products, Tuberin and Hamartin, control mTOR signaling by acting as a GTPase-activating protein complex toward Rheb. Curr Biol 13: 1259-1268, 2003. 
25. Bohensky J, Leshinsky S, Srinivas V and Shapiro IM: Chondrocyte autophagy is stimulated by HIF-1 dependent AMPK activation and mTOR suppression. Pediatr Nephrol 25: 633-642, 2010

26. Xue JF, Shi ZM,Zou J and Li XL: Inhibition of PI3K/AKT/mTOR signaling pathway promotes autophagy of articular chondrocytes and attenuates inflammatory response in rats with osteoarthritis. Biomed Pharmacother 89: 1252-1261,2017.

27. Takayama K, Kawakami Y, Kobayashi M, Greco N, Cummins JH, Matsushita T, Kuroda R, Kurosaka M, Fu FH and Huard J: Local intra-articular injection of rapamycin delays articular cartilage degeneration in a murine model of osteoarthritis. Arthritis Res Ther 16: 482, 2014.

28. Rosa SC, Rufino AT, Judas F, Tenreiro C, Lopes MC and Mendes AF: Expression and function of the insulin receptor in normal and osteoarthritic human chondrocytes: Modulation of anabolic gene expression, glucose transport and GLUT-1 content by insulin. Osteoarthritis Cartilage 19: 719-727, 2011.

29. Cravero JD, Carlson CS, Im HJ, Yammani RR, Long D and Loeser RF: Increased expression of the Akt/PKB inhibitor TRB3 in osteoarthritic chondrocytes inhibits insulin-like growth factor 1-mediated cell survival and proteoglycan synthesis. Arthritis Rheum 60: 492-500, 2009.
30. Hahn-Windgassen A, Nogueira V, Chen CC, Skeen JE, Sonenberg N and Hay N: Akt activates the mammalian target of rapamycin by regulating cellular ATP level and AMPK activity. J Biol Chem 280: 32081-32089, 2005.

31. Peng M, Huang Y, Tao T, Peng CY, Su Q, Xu W, Darko KO, Tao X and Yang X: Metformin and gefitinib cooperate to inhibit bladder cancer growth via both AMPK and EGFR pathways joining at Akt and Erk. Sci Rep 6: 28611, 2016.

32. Galasso O, Panza S, Santoro M, Goldring MB, Aquila S and Gasparini G: PTEN elevation, autophagy and metabolisc reprogramming may be induced in human chondrocytes during steroids or nutrient depletion and osteoarthritis. J Biol Regul Homeost Agents 29 (Suppl 4): S1-S14, 2015.

33. Memmott RM and Dennis PA: Akt-dependent and -independent mechanisms of mTOR regulation in cancer. Cell Signal 21: 656-664, 2009.

34. O'Reilly KE, Rojo F, She QB, Solit D, Mills GB, Smith D, Lane H, Hofmann F, Hicklin DJ, Ludwig DL, et al: mTOR inhibition induces upstream receptor tyrosine kinase signaling and activates Akt. Cancer Res 66: 1500-1508, 2006.

35. Yori JL, Lozada KL, Seachrist DD, Mosley JD, Abdul-Karim FW, Booth CN, Flask CA and Keri RA: Combined SFK/mTOR inhibition prevents rapamycin-induced feedback activation of AKT and elicits efficient tumor regression. Cancer Res 74: 4762-4771, 2014. 